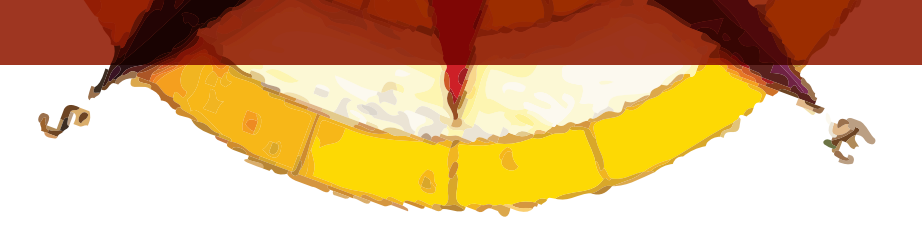

\title{
COTIDIANIDAD Y ENSEÑANZA GEOGRÁFICA
}

\author{
Alexánder Cely Rodríguez y Nubia Moreno Lache, \\ compiladores $^{90}$
}

\section{Uriel Coy Verano ${ }^{91}$}

Presentar un libro, escrito por profesores que han tenido la doble condición de ser mis profesores y por otro ser mis alumnos y ahora colegas tiene connotaciones especiales como quiera que se sea el puente entre dos generaciones. Para el caso de mis profesores, el libro al estar dedicado a la profesora Rosa Cecilia Torres de Cárdenas - Rosita - q.e.p.d., conlleva un sentimiento de gratitud, aprecio y valoración de su obra como docente y como persona que hoy extrañamos quienes la conocimos $\mathrm{y}$ fuimos sus alumnos.

Sus enseñanzas fueron fundamentales en nuestra formación, en nuestro desempeño profesional y en general en la vida misma; Rosita construyó y nos formó con la rigurosidad del conocimiento, con la búsqueda permanente del saber, con la mística y la entrega de lo que es y significa ser docente; contribuyo a través de sus enseñanzas y de su ejercicio docente e investigativo y uno de sus frutos es esta publicación. En ella se da cuenta de su trabajo, de sus enseñanzas, que evidenciamos en nuestros aprendizajes.

Una semblanza de la profesora Rosita, la hace la profesora Amanda de Moreno en la primera parte del texto y en la que señala el significado como compañera, jefe, colega y amiga. En fin, el lenguaje es corto para darle el verdadero sentido y la proporción de lo que Rosita significa para muchos de los docentes del país.

Veamos el libro: COTIDIANIDAD Y ENSEÑANZA GEOGRAFICA se organiza y presenta en dos partes; en la primera se destaca los aportes y realizaciones de algunos integrantes del grupo de investigación GEOPAIDEIA, quienes a través de los resultados de investigación destacan algunas de las diferentes líneas y proyectos que el grupo desarrolla y ha desarrollado en sus ya casi 15 años de vida. Como bien se señala en el prólogo, el libro es una compilación de materiales sobre la enseñanza en la geografía centrada en la reflexión pedagógica sobre algunos de sus problemáticas, no solo desde la geografía, sino de la pedagogía y la didáctica.

Las discusiones que se abordan, todas ellas acompañadas con una necesaria y justa reflexión teórica, soportan la rigurosidad que el proceso de investigación demanda, así como la posibilidad de

90 Cely, A., Moreno, N. (2008) Cotidianidad y enseñanza geográfica. Bogotá D.C.: Editorial Códice

91 Docente Universidad Distrital Francisco José de Caldas. 
interactuar con el medio y con los sujetos que construyen espacio. Pero así mismo, con la capacidad propositiva y crítica no solo para generar nuevos aportes metodológicos, sino también para avanzar en la construcción y formulación de una didáctica de la geografía más pertinente con el entorno y con la realidad de los ciudadanos.

Asumen los autores, de entrada, categorías que tranversalizan los contenidos del libro: el valor de la vida cotidiana, el sentido de la observación, la percepción del ciudadano, los espacios significativos, el significado del territorio para los sujetos que construyen espacio, entre otros. Lo anterior esta mediado con una rigurosa observación y adaptación de la realidad, dan y argumentan afirmaciones y propuestas con la solidez y rigurosidad que soporta la lectura y la critica que el mundo académico le exige a sus actores.

Tal como se señala en la introducción, el libro se centra en los maestros y estudiantes y es transversalizado por el espacio geográfico, es decir, es la deliberación de profesores y estudiantes sobre su propia geografía.

Papel fundamental en la reflexión lo desarrolla la Maestría en Educación con Énfasis en Docencia de la Geografía; si bien al grupo de investigación es quien articula e integra, fueron los desarrollos de la Maestría con sus profesores los que orientan y construyen las líneas básicas del desarrollo curricular y de investigación que la sustenta. Pero así mismo lo son los estudiantes quienes a través de su ejercicio profesional - porque todos son maestros -, y en su condición de estudiante-investigador, porque esta debe ser una condición que toda Maestría debe promover, y ellos, aplicando enseñanzas, y sujetos de sus propios aprendizajes supieron plasmar en la investigación; es decir, los conocimientos geográficos, pedagógicos y didácticos que son la impronta de la Maestría.

La Maestría es una apuesta para impactar, transformando la enseñanza y el aprendizaje de la geografía desde las primeras fases de formación del ciudadano. Se trata de superar posturas rígidas, atadas a viejas concepciones tanto del saber geográfico, pedagógico y didáctico a otras quizás más modernas, menos rígidas, que valoran y le dan sentido al espacio como una construcción social en el que los sujetos sociales aportan desde sus propias realidades, entornos y significados a lo que es la geografía y/o su didáctica.

Independiente de la aproximación a conceptos básicos del saber geográfico, la definición de posturas pedagógicas y didácticas y su incorporación en los procesos de aprendizaje y de investigación permiten que con rigurosidad y autoridad, se formulen proposiciones y se hacen recomendaciones para transformar las prácticas del aprendizaje de la geografía con la responsabilidad que le compete a las instituciones, a los responsables de la enseñanza y a los estudiantes: en el primer caso, con la incorporación de otros elementos en los currículos y programas en donde forman profesores y, en últimas, promoviendo las orientaciones para generar las transformaciones necesarias. En los maestros para modificar líneas y formas de pensamiento sobre el saber geográfico y sus didácticas y en los estudiantes porque recrean los aprendizajes para aprender a actuar y transformar su propio espacio. 


\section{UNA MIRADA A LOS CONTENIDOS DEL LIBRO}

\section{Bogotá con ojos de taxista \\ Elsa Amanda Rodríguez de Moreno y Alexandra Von Pral. Ramírez}

Las autoras presentan un escrito que recogiendo las vivencias y vidas de un grupo de taxistas, dan cuenta del potencial que tiene la ciudad para su aprendizaje, gracias a sus imaginarios y experiencias de vida, en el diario vivir, en la cotidianidad del trashumar de la calle. Los taxistas construyen sus concepciones de espacio, tiempo y lugar, definen sus propias toponimias, precisan categorías de espacio, crean sus propios hitos, construyen los senderos y rutas y le dan el sentido a su propio espacio urbano.

Hacen las autoras un esfuerzo por identificar a partir de la percepción, la subjetividad y el comportamiento una caracterización de sus diversas ciudades, destacando la singularidad de cada una de ellos, aun corriendo el riesgo de terminar negando la integralidad del espacio.

En el escrito se tratan de generar respuestas de cómo los actores de ciudad, en este caso los taxistas, piensan espacialmente la ciudad, como conocen el espacio geográfico, identifican las relaciones topológicas y sus formas de representación, las que a través de una cartografía social hacen o logran construir sus representaciones sociales, es decir, grafican sus propias percepciones estableciendo, o mejor permitiendo identificar la necesaria relación entre el conocimiento de la cotidianidad con conceptos y teorías que sustentan el espacio. Se aprecia un esfuerzo en las autoras, que hay que reconocer y destacar, por esos conceptos, formas, categorías y topologías de espacios urbanos. Pero también la diferenciación del espacio en donde las dinámicas propias de la cotidianidad, son interpretadas para cumplir con los propósitos planteados en los objetivos de la investigación. De ahí que en las conclusiones se validó destacar el carácter dinámico diferenciado, heterogéneo y concreto que forman parte del espacio geográfico.

\section{Geografia y Literartura: Una alternativa para la enseñanza y comprensión del espacio geográfico}

\section{Alexander Cely Rodriguez y Nubia Moreno Lache}

El artículo desarrolla una temática interesante y necesaria que cubre un amplio espectro de la cultura. Los autores abordan la reflexión de la literatura y si bien lo hacen a través de una de las expresiones clásicas como es la novela, es de entender que la literatura tiene otras manifestaciones vinculado con la poética, el ensayo, la crónica, el poema, entre otras, que no son consideradas. Y, qué decir de la incursión en otras expresiones de la cultura como lo son la música el cine, para mencionar solamente estas. Aquí no se trata de problematizar a los autores con nuevas tareas, sino de llamar la atención sobre la diversidad de la cultura en el proceso de darle sentido y significado a lo urbano desde otras ópticas.

Pero, y como bien lo señalan los autores, en la obra literaria se articula no solamente la percepción, sino también la imaginación y hasta la utopía, sobre el espacio geográfico, en este caso el urbano. Así, la literatura permite reflejar por una parte los aportes que interpretando la realidad o la subjetividad misma del escritor presentan la realidad urbana, independiente de los riesgos de la subjetividad y del lenguaje del escritor en su escrito, y/o de la capacidad del lector para interpretarla 
y darle sentido a las palabras, o del mensaje que se quiere generar. La anterior controversia, reviste especial importancia en tanto podría quitarle objetividad a la realidad que es objeto de la obra literaria, no sin antes valorar el que "conocer y comprender otras realidades y mundos posibles a través de la literatura, motiva una enseñanza y un aprendizaje de la geografía y de los conocimientos acordes y pertinentes con las demandas socio - culturales de los sujetos y de las subjetividades."

"Esta condición valida los diversos esfuerzos existentes por conocer, comprender y explicar el espacio ocupado por el hombre y expresado desde la relación individuo - comunidad; de ahí que se rescata la literatura como saber que sustenta estrategias de aprendizaje de la geografía y, en particular, de la geografía urbana a partir de la vivencia subjetiva.", y que los autores plasman perfectamente en la obra.

En cualquier caso es importante distinguir las tres perspectivas que los autores citan para aproximarnos a la relación ciudad y educación: aprender en la ciudad, aprender de la ciudad, aprender la ciudad (Trilla; 1990) y al que agregaríamos aprender con la ciudad como quiera que somos constructores de ciudad.

Por otra parte, en la argumentación de las vivencias y aportes de la literatura al conocimiento del hecho urbano, los autores formulan unos procesos de investigación que partiendo de la redefinición conceptual avanza en la identificación de sitios y lugares, del análisis tanto en la obra que se analiza, como el autor, con las vivencias subjetivas de los lectores hasta las categorías espaciales y la identificación de conceptos con los que se escribe, lee e interpreta la obra literaria.

Lo anterior destaca el hecho planamente reconocido de las relaciones entre literatura y ciudad, y del valor de la primera para poder darle significado a lugares y formas y, sobre todo para entender que la literatura como expresión de la cultura, refleja y le da sentido desde la palabra a los hechos que construyeron espacio, es decir, le permite a los lectores proyectarse en los hechos sociales que construyeron o coadyudaron a la construcción de un determinado espacio.

\section{La investigación - Acción en la enseñanza y el apredizaje de la geografía Alix Otalora Duran}

El articulo producto de un proyecto de investigación del grupo de GEOPAIDEIA, establece una relación sobre la enseñanza y el aprendizaje de la geografía con la practica pedagógica, destacando su importancia en la formación del futuro educador. Para ello aborda temáticas que van desde la teorización y los aspectos metodológicos y didácticos, hasta la formulación de una propuesta de formación de docentes.

Recupera y defiende los postulados de la investigación -acción como baluarte del proceso de investigación, denominados en muchos de los apartes como proyecto pedagógico - investigativo, y que en últimas reflejan la experiencia y las vivencias del estudiante en su práctica pedagógica.

En el texto el proyecto pedagógico investigativo, que se desarrolla en tres fases, incluye elementos asociados con la contextualización y caracterización del entorno de los estudiantes, la implementación, seguimiento y evaluación de una propuesta de intervención didáctica, y culmina con la sistematización y evaluación del proyecto pedagógico. 
Como es característica general en todos los documentos del libro, y siguiendo las líneas de orientación metodología para la investigación, el texto se acompaña de una reflexión teórica inicial, una fase de trabajo de campo y captura de información que es sistematizada en fichas, y avanza en la caracterización de las realidades geográficas, pedagógicas y didácticas en donde el estudiante desarrolla su proyecto pedagógico investigativo.

Un valor agregado al aporte innovador de la investigación - acción con todos sus componentes, procedimientos e instrumentos lo que constituye un reto para la Universidad y el Departamento de Ciencias Sociales de la Universidad Pedagógica Nacional para asumir los procesos de transformación en la formación de los futuros docentes que requiere el país.

\section{La salida de campo. una manera de enseñar y aprender geografía Ana Griselda Perez y Liliana Angelica Rodríguez}

En el artículo las colegas abordan la salida de campo como una estrategia de aprendizaje de la geografía, para lo cual se parte de una conceptualización del espacio geográfico como objeto de aprendizaje a saber: se soporta en relatos de dos experiencias de campo en diferentes sectores de la ciudad y en diferentes momentos (día y noche).

Independiente de la valoración como estrategia didáctica, las autoras proponen unos elementos denominados que son ámbitos de intervención para abordar diferentes intencionalidades y/o componentes de la estructura espacial.

Las actividades del trabajo de campo incluyen actividades previas, durante y después de la realización de la actividad. Si bien muchos de los elementos se centran en los registros de observación, imágenes, apropiación y elaboración de cartografía, le permiten al estudiante generar aprendizajes y aprehensión del espacio no solo desde el contenido y organización de los componentes de la estructura espacial, sino desde la relación y diferenciación espacial propia de las formas espaciales, sabiamente conceptualizadas por Milton Santos.

Los tres textos anteriores y siguiendo algunos de los planteamientos que subyacente en los desarrollos de la ciudad educadora, bien se pueden equiparar con algunas estrategias didácticas en el marco de lo que algunos denominamos las inmersiones geográficas.

\section{Globalización en el aula de clases. una experiencia de aula Carlos Alberto Zambrano}

El profesor Zambrano inicia su disertación planteando algunos cambios entre la enseñanza tradicional y las nuevas formas de enseñar, esta última orientada fundamentalmente al aprendizaje y la autonomía de los estudiantes, sin que ello signifique que el docente renuncie a su deber de orientar los procesos de enseñanza y por consiguiente el resultado del aprendizaje de sus alumnos.

El pretexto para generar ese cambio paradigmático es el tema de la globalización como tema de reflexión y discusión en el aula de clase. El autor parte de diferenciar los conceptos de internacionalización y mundialización, como pasos necesarios en lo que se puede llamar "una didáctica de la globalización". Esta didáctica de la globalización pasa por asumir las categorías de estructura, es- 
tadio, coyuntura y proceso que son connaturales a todo proceso de globalización, según lo sostiene y desarrolla el autor.

Haciendo uso de dinámicas de grupos, juegos, debates, mesas redondas, seminarios, entre otras, desarrolla con sus estudiantes en el aula, actividades que variando desde la preparación desarrollo y la retroalimentación, se trabaja en el progreso de una temática específica, en este caso, la globalización.

Cada una de las etapas conllevan fases previas de revisión de documentos que permiten la caracterización de territorios -objeto de la globalización- a través de investigaciones de fuentes secundarias y en general acopio de información, que sirven de base para construir y llegar a unas conclusiones que serán objeto de discusión y acuerdos por parte de los estudiantes.

La sistematización de resultados, consensos, acuerdos hasta llegar a conclusiones, le otorgan un valor agregado al trabajo, lo que se complementaria, con desarrollar competencias en comunicación, no solamente oral si no escrita y de enorme importancia en los procesos de formación de nuestros estudiantes.

La segunda parte del libro titulado EDUCACIÓN Y GEOGRAFÍA, EXPERIENCIAS SUGERENTES DE INVESTIGACIONES EL AULA, es desarrollada por los profesores Elsa Amanda Rodríguez de Moreno, Rosa Cecilia Torres de Cárdenas, Maria Cristina Franco Arbelaez y Gustavo Montañez Gómez.

Esta parte del libro presenta las concepciones, procedimientos y resultados de la Maestría en Educación con Énfasis en Docencia de la Geografía que ofrece la Universidad Pedagógica Nacional. Los autores desarrollan la temática; los conceptos y la didáctica; el espacio geográfico; un diagnóstico sobre nociones y manejos de conceptos sobre el espacio geográfico; la Maestría en Educación con énfasis en Docencia de la Geografía: Una posibilidad para la praxis, y la propuesta para la enseñanza de la geografía en la educación básica

\section{LOS CONCEPTOS Y LA DIDÁCTICA}

Los apartes del texto inician haciendo unas precisiones respecto al saber geográfico y las concepciones que desde la pedagogía se están desarrollando. Como en un caso anterior, los planteamientos apuntan a modificar ciertas prácticas pedagógicas de carácter tradicional, asumiendo el constructivismo como una de las nuevas pedagogías.

La reflexión que desarrollan los autores se fundamenta en que "Los fundamentos de una pedagogía constructivista no son solo complejos si no que su aplicación implica un vasto conjunto de tareas. Entre otras, se debe tratar de cambiar la estructura cognoscitiva que tienen los mismos investigadores en relación con los conceptos de pedagogía, didáctica y geografía, siendo estas quizás, la primera y la fundamental".

Así mismo, se argumenta que los aprendizajes deben estar basados en estrategias que permitan diagnosticar la construcción de concepto de espacio geográfico, como vía inicial de reestructuración, de tal manera que ésta asuma una concepción de constructivismo dinámico y no estático. 
Se propone que un mecanismo para desarrollar propuesta desde el constructivismo son los mapas conceptuales ya que en ellos se explicitan los conceptos, sus relaciones e interrelaciones y facilita niveles de jerarquización.

El texto hace una revisión de algunas definiciones sobre la didáctica, concluyendo que se debe incluir la reflexión sobre la enseñanza y el aprendizaje teniendo en cuenta las condiciones del entorno y las características cognoscitivas, sociales y afectivas de los alumnos. Lo anterior conlleva que la didáctica debe ser redefinida y descontextualizada para facilitar el aprendizaje de los alumnos, permitiendo de paso, lograr aprendizajes significativos y por consiguiente construir contenidos geográficos pertinentes.

Así mismo para los autores, es importante que se abandonen posturas didácticas que privilegien un enfoque geográfico en particular y que le permita al estudiante construir sus propios conceptos geográficos. El trabajo del docente debe orientarse a facilitar y dilucidar los conceptos del saber geográfico específico según el entorno y las condiciones particulares de los estudiantes.

\section{ESPACIO GEOGRÁFICO}

Los autores reconocen los niveles de complejidad asociados al concepto de espacio geográfico, independiente que desde la geografía se generen avances significativos. Se propone que la pedagogía contribuya a la construcción del concepto, principalmente en el ámbito escolar. El proceso pedagógico requiere de la realización de actividades vivenciales, perceptuales y conceptúales con niveles de sistematización y organización que den coherencia al concepto.

Los conceptos deben ser concebidos como un sistema integrado de tres grandes grupos denominadas acciones, dones naturales y sistemas de objetos. Esa cualificación conlleva que se superen la dualidad entre lo físico y lo natural y que se avance en un concepto integral y más armonioso del espacio geográfico, todos ellos orientados a la explicación y comprensión del propio espacio.

Así se tiene que "El reto pedagógico implica el diseño, planeación y realización de un proceso de enseñanza - aprendizaje que posibilite el desarrollo de capacidades conceptuales y cognitivas para que los estudiantes puedan construir el concepto de espacio geográfico y demostrar sus capacidades de análisis y síntesis para comprenderlo y explicarlo."

\section{DIAGNÓSTICO SOBRE NOCIONES Y MANEJOS DE CONCEPTOS SOBRE EL ESPACIO GEOGRÁFICO}

La tercera parte de este segmento del libro es un desarrollo de las aplicaciones conceptuales alrededor de la didáctica y el espacio geográfico que se reflejan en espacios de investigación desarrollados por los estudiantes de la Maestría.

Las cinco experiencias presentadas, tratan de seguir un patrón similar en la investigación: una primera donde se profundizan conceptos básicos, una segunda en donde se escriben las herramientas de orden metodológico y, una tercera que además de presentar los resultados y de los hallazgos se plantean conclusiones que en muchos de los casos se convierten en recomendaciones para transformar conceptos o para introducir nuevas estrategias didácticas y/o para profundizar en nuevos estudios. 
Si bien en todos los casos y siguiendo los lineamientos iniciales se hace una caracterización de los entornos de las instituciones y de la comunidades que sirvieron de basa para adelantar la investigación, en todos los casos el trabajo en el aula y en el campo con los estudiantes caracterizan el trabajo desarrollado en las investigaciones trabajadas.

Es de anotar en cada una de las experiencias se abordaron temáticas deferentes que varían desde las nociones de espacio geográfico en áreas urbanas a nociones de espacio en áreas rurales, representaciones graficas del espacio, concepciones geográficas de alumnos y concepciones de espacio geográfico de profesores. Hay una clara intencionalidad de los autores de suministrar elementos de contraste, ejemplos y realizaciones que permiten valorar positivamente el trabajo realizado.

Con todo, la complejidad de la temática que significan los nuevos aprendizajes de la geografía lleva a pensar que independiente de los trabajos de grado realizados y su caracterización en las diferentes cortes, reclaman la urgencia de su continuidad. Muchos tópicos, entornos, aspectos teóricos tanto de la geografía como de la didáctica requieren más investigaciones.

Por otra parte, es importante introducir otras concepciones pedagógicas y didácticas como quiera que al asumir una sola tendencia pedagógica, fácilmente permite caer en el unanimismo y concepciones excluyentes que niegan el carácter etéreo y complejo tanto de la geografía y del espacio: es una tarea que a mi modo de ver debe ser liderada por las Facultades de Ciencias y de Educación, independiente de las propuestas que para la enseñanza de la geografía en educación básica, los autores sugieren en el apartado quinto de esta parte del libro.

En conclusión, una interesante y necesaria publicación que es ineludible leer y releer, digerir y aprovechar por quienes hacemos de su objeto de vida formar ciudadanos comprometidos con su territorio y con su entorno espacial.

Artículo recibido 21 - 09 - 10. Aprobado 26 - 11 - 10. 